\title{
On Deriving the Upper Bound of $\alpha$-Lifetime for Large Sensor Networks
}

\author{
Honghai Zhang and Jennifer Hou \\ Department of Computer Science \\ University of Illinois at Urbana-Champaign \\ Email:\{hzhang3,jhou\}@cs.uiuc.edu
}

\begin{abstract}
In this paper, we explore the fundamental limits of sensor network lifetime that all algorithms can possibly achieve. Specifically, under the assumptions that nodes are deployed as a Poisson point process with density $\lambda$ in a square region with side length $\ell$ and each sensor can cover a unit-area disk, we first derive the necessary and sufficient condition of the node density in order to maintain complete $k$-coverage with probability approaching 1 . With this result, we obtain that if $\lambda=\log \ell^{2}+(k+2) \log \log \ell^{2}+c(\ell), c(\ell) \rightarrow-\infty$, as $\ell \rightarrow+\infty$, the sensor network lifetime (for maintaining complete coverage) is upper bounded by $k T$ with probability approaching 1 as $\ell \rightarrow+\infty$, where $T$ is the lifetime of each sensor. Second, we derive, given a fixed node density in a finite (but reasonably large) region, the upper bounds of lifetime when only $\alpha$-portion of the region is required to be covered at any time. We also carry out simulations to validate the derived results. Simulation results indicate that the derived upper bounds apply not only to networks of large areas but also to small-area networks.
\end{abstract}

\section{Categories and Subject Descriptors} C.2.1 [COMPUTER-COMMUNICATION NETWORKS Network Architecture and Design-Wireless Communication; G.3 [Probability and Statistics]: Stochastic processes

\section{General Terms}

Performance, Reliability, Theory

\section{Keywords}

Poisson point process, coverage, k-coverage, lifetime, sensor networks, fundamental limits

\section{INTRODUCTION}

Driven by advances in MEMS micro-sensors, wireless networking, and embedded processing, ad-hoc networks of sen-

Permission to make digital or hard copies of all or part of this work for personal or classroom use is granted without fee provided that copies are not made or distributed for profit or commercial advantage and that copies bear this notice and the full citation on the first page. To copy otherwise, to republish, to post on servers or to redistribute to lists, requires prior specific permission and/or a fee.

MobiHoc'04, May 24-26, 2004, Roppongi, Japan.

Copyright 2004 ACM 1-58113-849-0/04/0005 ...\$5.00. sors have become increasingly available for commercial and military applications such as environmental monitoring (e.g., traffic, habitat, and security), industrial sensing and diagnostics (e.g., factory and appliances), monitoring critical infrastructures (e.g., power grids, water distribution and waste disposal), and information collecting for battlefield awareness $[1,8,13,14]$.

Sensor nodes in such a network usually have limited onboard processing and wireless communication capabilities, and are equipped with batteries with limited power. Moreover, it is impractical or infeasible to replenish energy via replacing batteries on these sensors in most applications. As a result, it is well perceived that a sensor network should be deployed with reasonable density (up to 20 nodes $/ \mathrm{m}^{3}$ [18]) in order to prolong the network lifetime.

In a high-density network with energy-constrained sensors, it is neither necessary nor desirable to have all nodes operate simultaneously in the active mode. Density control - the function that controls the density of working sensors at a desirable level — becomes an important issue [22]. Specifically, density control ensures only a subset of sensor nodes operate in the active mode, while meeting the following two requirements: (i) coverage: the region that can : be monitored is not smaller than the region that can be monitored by a full set of sensors; and (ii) connectivity: the sensor network remains connected so that the information collected by sensor nodes can be relayed back to data sinks or controllers. In addition, to prolong network lifetime, it is desirable that nodes wake up on a rotational basis and at any time only a minimum set of sensors wakes up to maintain coverage and connectivity. Several authors have proposed several algorithms for this purpose [23, 19, 25, 10, 20].

In this paper, instead of proposing another algorithm for selecting the set of working sensor nodes, we explore the fundamental limit of sensor network lifetime that all algorithms can possibly achieve. The derivation is based on the theory of coverage processes [11] and made under the assumptions that the locations of the deployed sensors form a Poisson point process in a square region and that sensor nodes only fail because of power depletion (but not of malicious destruction). First, we prove that a necessary and sufficient condition of complete $k$-coverage of a square region with side length $\ell$ (in the almost surely sense) is the density of the nodes $\lambda=\log \ell^{2}+(k+1) \log \log \ell^{2}+c(\ell)$ where $c(\ell) \rightarrow+\infty$ as $\ell \rightarrow \infty$. And consequently, given the density $\lambda=\log \ell^{2}+(k+2) \log \log \ell^{2}+c(\ell)$ and $c(\ell) \rightarrow-\infty$, the lifetime upper bound is $k T$ with probability approaching 1 as $\ell \rightarrow+\infty$, where $T$ is a single sensor lifetime. Second, 
we derive, given a certain density in a finite (but still large) region, the upper bounds of the lifetime under the scenario that only $\alpha$-portion of the region is required to be covered. In this scenario, we derive two upper bounds: one holds universally for any possible algorithm, and the other is targeted for algorithms that attempt to completely cover the region initially and gradually reduce the coverage ratio, until it drops below a certain threshold $\alpha$.

We also carry out a simulation study to validate the derived upper bounds of network lifetime under sensor networks within reasonably large areas. Simulation results indicate that the derived upper bounds apply not only to sensor networks within large areas, but also to networks of small areas, although in the latter case the derived upper bounds may not be tight.

With our derivation and simulation results, we will be able to answer several important questions, e.g., given the lifetime $T$ of a single sensor node, how many sensor nodes (or the sensor density) have to be deployed in a region, in order to continuously monitor the region for a period of $k \cdot T$. We also observe that although it is, in general, desirable to deploy a sensor network of high density to achieve a large lifetime per unit of nodal density, the increase in the lifetime per unit of nodal density becomes marginal when the density exceeds certain threshold. The overhead incurred in maintaining coverage in a distributed manner dominates when the sensor density becomes high.

Several research efforts have been made to derive the upper bounds of network lifetime in wireless ad hoc networks and sensor networks $[3,4,5]$. We will give a detailed summary of existing work in Section 2. Our work is different from existing works in two aspects. First, we consider as the network lifetime the time interval during which at least $\alpha$-portion of the region can be continuously monitored. Second, the lifetime upper bounds derived in this paper are independent of power-saving schemes used.

The rest of the paper is organized as follows. In the next section, we summarize the related work. Section 3, we state the assumptions we make on the system model, define what we mean by network lifetime in sensor networks. Then we present our derivation in Section 4 and 5. Following that, we carry out simulations in Section 6 to validate the derived results. Finally we conclude the paper in Section 7 .

\section{RELATED WORK}

Several researchers $[22,23,19,25,10,20]$ have addressed various methods of minimizing energy consumption and prolonging network lifetime while maintaining coverage and connectivity in wireless sensor networks.

Ye et al. [22, 23] present PEAS, a distributed, probingbased density control algorithm for robust sensing coverage. In this work, a subset of nodes operate in the active mode to maintain coverage while others are put into sleep. It ensures no two active nodes are in the proximity of each other but does not preserve complete coverage.

Tian et al. [19] devise an algorithm that ensures complete coverage using the concept of "sponsored area." Whenever a sensor node receives a packet from one of its working neighbors, it calculates its sponsored area (defined as the maximal sector covered by the neighbor). If the union of all the sponsored areas of a sensor node covers the coverage disk of the node, the node turns itself off.

Zhang and Hou [25] analyze the relationship between com- plete coverage and connectivity, develop some optimal conditions of maintaining coverage, and devise a localized method to maintain coverage and connectivity based on the optimal conditions.

Wang et al. [20] analyze the relationship between $k$ coverage and $j$-connectivity, prove a sufficient condition for satisfying $k$-coverage and propose an algorithm (combined with SPAN [6]) to maintaining coverage and connectivity based on the sufficient condition.

Gupta et al. [10] devise both a centralized and a distributed algorithm to find a subset of nodes that ensure both coverage and connectivity. The centralized algorithm guarantees that the size of the formed subset is within $O(\log n)$ factor of the optimal size, where $n$ is the network size.

Although all the above methods are targeted for prolonging the network lifetime, most of them do not perform any analysis on the network lifetime. Recently, research efforts have also been made to analyze the upper bound of the lifetime for ad hoc and/or sensor networks [3, 4, 5, 7]. Bhardwaj et al. [3, 4] study the upper bound of the lifetime of data gathering sensor networks. They assume the data source is randomly distributed in a region with a certain p.d.f function and the data sink is located at a fixed point. They calculate the minimum power required to transmit a bit from the source to the sink and then compute the upper bound of the network lifetime based on the minimum power consumption. In [4] they do not consider network topology or the effect of data aggregation of data streams. In [3] they extend the work in [4] by taking into account of these factors and deriving the upper bound of the network lifetime for networks with arbitrarily complex capabilities. However, their model only considers the power consumption when sensor nodes sense active events, process, transmit and/or receive data, but not when sensor nodes are monitoring but sense no active events. As shown in the empirical study in $[18,9]$, energy is consumed not only by active communications, but also by wireless devices in the idle and/or sensing state. As a matter of fact, the energy consumed by wireless devices in the idle and/or monitoring state is only a little less than that in the transmitting or receiving states. Thus it makes more sense to derive the network lifetime under the scenario that only a minimum set of sensors are turned on, while the other sensors operate in the low-power mode (or sleep mode).

Blough and Santi [5] study the upper bound of the network lifetime for cell-based energy conservation techniques. While the bound derived does consider energy consumption both in the transmitting/receiving state and in the idle state, it is restricted to the GAF scheme proposed in [21]. In contrast, the lifetime derived in this paper is independent of power-saving schemes used.

Coleri et al. [7] investigate the lifetime of networked sensor nodes where sensors are organized in a tree-based multihop networks. They analyze the lifetime of nodes in four different groups based on their distances to the data sink using the finite automata technique. However, their analysis is primarily on the lifetime of individual nodes instead of that of the network.

\section{PRELIMINARY}

To facilitate the derivation, in this section we state the assumptions we make on the system model, and define the network lifetime in sensor networks. 


\subsection{Assumptions on the System Model}

We assume the region $R$ to be monitored is a square region with side length $\ell$. We further assume torus convention (a.k.a. the toroidal model [15]) described in [11], page 23, i.e., each disk that protrudes one side of the region $R$ enters $R$ again from the opposite side (Fig. 1). This eliminates consideration of boundary effects. Each sensor node can detect an event of interest within a distance of $r$, and this distance is termed as the sensing range. The disk centered at a sensor node and with a radius of $r$ is termed as the coverage disk of this node. Without loss of generality, we assume that each sensor node has a sensing range of $r=\frac{1}{\sqrt{\pi}}$ and thus each sensor node can cover a disk of unit area. We assume $\ell>>r$. We assume that each sensor has the same lifetime of $T$. This assumption is generally made when to analyze the network lifetime, for example, in [5].

We assume the deployed sensor nodes in the square region $R$ form a (homogeneous) Poisson point process with density $\lambda$. There are several ways of defining a Poisson point process, one of which is stated below. First, for any subset $A$ of the region $R$, the distribution of the number of nodes in the set is Poisson with mean $\lambda\|A\|$, where $\|A\|$ is the area of $A$. Second, given that the number of nodes in such a set $A$ is $m$, the node locations in $A$ are $m$ mutually independent random variables, each uniformly distributed over $A$. It is well known that $n$ nodes whose locations are mutually independent random variables, each with uniform distribution in $R$, are essentially a Poisson point process with density $\lambda=n / \ell^{2}$ if $R$ is large ([11], page 39).

As analytically proved in [25], if the radio transmission range is at least twice as large as the sensing range, network coverage implies connectivity. That is, as long as the set of working nodes completely covers the monitored region, the network is connected. We make this assumption so as to facilitate derivation. As tabulated in Tables 1-2, this assumption holds for most commercially available sensor devices. A study on the network lifetime when the above assumption does not hold (and hence one has to consider both coverage and connectivity in selecting the working set) is a subject of future investigation.

\subsection{Definition of Sensor Network Lifetime}

We define the $\alpha$-lifetime as the entire interval in which at least $\alpha$ portion of the region $R$ is covered by at least one sensor node, where $\alpha$ is a tunable parameter.

A discussion on how the $\alpha$-lifetime defined above compares against the lifetime defined in [5] and [22] is in order. Blough and Santi [5] defined the lifetime of sensor networks as $\min \left\{t_{1}, t_{2}, t_{3}\right\}$, where $t_{1}$ is the time it takes for the cardinality of the largest connected components to drop below $c_{1} \cdot n(t)$, where $n(t)$ is the number of alive nodes at time $t$, $t_{2}$ is the time it takes for $n(t)$ to drop below $c_{2} \cdot n(0)$, and $t_{3}$ is the time it takes for the area covered to drop below $c_{3} \cdot \ell^{2}$. Here $0 \leq c_{1}, c_{2}, c_{3} \leq 1$. If we set $c_{1}=0, c_{2}=0$ and $c_{3}=\alpha$, then the network lifetime is exactly the same as the $\alpha$-lifetime defined in this paper. Under the assumption that the radio range is at least twice as large as the sensing range (and thus network coverage implies connectivity), it makes sense to ignore the connectivity requirement imposed by $t_{1}$ and set $c_{1}=0$. The requirement imposed by $t_{2}$ is not really necessary in sensor networks, since one is usually not concerned with how many sensors remain alive but with whether or not the remaining sensors can perform certain

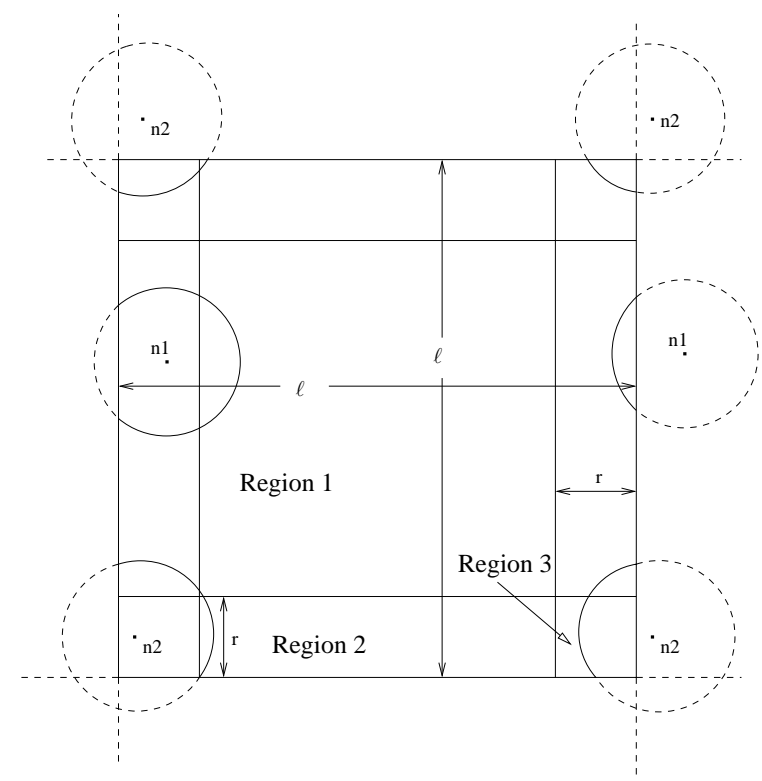

Figure 1: The toroidal model. The model can be interpreted by considering $R$ as simply one member of a lattice of squares and assuming that all nodes are repeated in precisely the same relative positions in all squares.

functions such as monitoring and relaying information back to data sinks.

Ye et al. [22] defined the lifetime as the time it takes for the coverage (defined as the ratio of the area covered by working nodes to the total area) to drop below, and never exceed again a pre-determined threshold. Due to the network dynamics, the coverage may occasionally drop below a threshold and come back again. They take into account of the time interval when the coverage temporarily drops below the threshold in the network lifetime, while we do not.

\section{ASYMPTOTIC UPPER BOUND OF 1-LIFETIME}

In this section, we investigate the asymptotic lower bound on the density $\lambda$ required to guarantee full coverage $(\alpha=1)$ for time $k T$ as the monitored region $\ell \rightarrow+\infty$. This result can also be interpreted as the asymptotic 1-lifetime upper bound given the density $\lambda$ of the sensor nodes. For completeness of the paper, we succinctly summarize several results of coverage processes [11] that pertain to our derivation in Appendix A.

The problem of deriving the upper bound of the asymptotic 1-lifetime is highly related to the $k$-coverage problem, where by $k$-coverage we mean every point in the monitored region is covered by at least $k$ nodes. Let the coverage number denote the maximum value of $k$ such that the sensor network has $k$-coverage in the monitored region. It is obvious that the coverage number $k$ times the lifetime $T$ of a single sensor gives a strict upper bound of the sensor network 1-lifetime.

Let the $k$-vacancy $V_{k}$ denote the area that is covered by at most $k-1$ nodes. We need to determine the required density $\lambda$ such that $P\left(V_{k}>0\right) \rightarrow 0$ as $\ell \rightarrow+\infty$. Let $\chi_{k}(x)$ denote 
the indication function of whether a point $x$ is covered by at most $k-1$ sensor nodes, i.e.,

$\chi_{k}(x)= \begin{cases}1, & \text { if at most } k-1 \text { nodes cover the point } x \\ 0, & \text { otherwise }\end{cases}$

The fact that a point $x$ is covered by at most $k-1$ sensor nodes indicates that there are at most $k-1$ sensor nodes within the unit-area disk centered at $x$ (recall that each sensor can cover a unit-area disk centered at itself). Under the assumption that the deployed sensors form a Poisson point process, we have

$$
P\left(\chi_{k}(x)=1\right)=e^{-\lambda}\left(\sum_{i=0}^{k-1} \frac{\lambda^{i}}{i !}\right) .
$$

Now the random variable $V_{k}$ can be expressed as

$$
V_{k}=\int_{R} \chi_{k}(x) d x
$$

To calculate its expectation, we use Fubini's theorem [16] and exchange the order of integral and expectation, i.e.,

$$
\begin{aligned}
E\left(V_{k}\right) & =\int_{R} E\left(\chi_{k}(x)\right) d x \\
& =\int_{R} P\left(\chi_{k}(x)=1\right) d x \\
& =\|R\| P\left(\chi_{k}(x)=1\right) \\
& =\ell^{2} e^{-\lambda}\left(\sum_{i=0}^{k-1} \frac{\lambda^{i}}{i !}\right),
\end{aligned}
$$

where the third equality results from the fact that $P\left(\chi_{k}(x)=\right.$ 1 ) is a constant for all $x$.

In order to ensure complete coverage for the duration of $k T$, each point should be covered by at least $k$ nodes, which implies $V_{k}=0$. As nodes form a Poisson point process in the region $R$, it cannot be guaranteed that this always occurs with a finite density $\lambda$, no matter how large $\lambda$ is. However, with $\lambda \rightarrow+\infty$ as $\ell \rightarrow+\infty$ we can ensure this occurs almost surely, i.e. $P\left(V_{k}=0\right) \rightarrow 1$ as $\ell \rightarrow+\infty$. This ensures complete $k$-coverage almost surely (see Remark 3 after the proof of the theorem below).

In what follows, we establish a tight bound on the density $\lambda$ that ensures asymptotic complete $k$-coverage.

TheOREM 1. Let $\lambda=\log \ell^{2}+(k+1) \log \log \ell^{2}+c(\ell)$. If $c(\ell) \rightarrow+\infty$ as $\ell \rightarrow+\infty$, then $P\left(V_{k}>0\right) \rightarrow 0$. If $c(\ell) \leq$ $C<+\infty$, then $P\left(V_{k}>0\right) \geq \epsilon$ as $\ell \rightarrow+\infty$, where $\epsilon=$ $1 /\left(1+4 e^{C}(k+1) !\right)$.

Proof. First we prove if $c(\ell) \rightarrow+\infty$ as $\ell \rightarrow+\infty, P\left(V_{k}>\right.$ $0) \rightarrow 0$. Clearly if the value of $\lambda$ increases, $P\left(V_{k}>0\right)$ will decrease. Hence we assume that $c(\ell)=o\left(\log \ell^{2}\right)$.

Define a crossing to be either an intersection point of the boundaries of two disks or an intersection point of the boundary of a disk and the boundary of region $R$. A crossing is said to be $k$-covered if it is an interior point of at least $k$ disks. By Theorem 4 in [20], region $R$ is completely $k$-covered if there exist crossing points and every crossing point is $k$-covered. Equivalently, if $R$ is not completely $k$ covered and there exist crossings, some of the crossings are not $k$-covered.

With $\lambda \rightarrow+\infty$ as $\ell \rightarrow+\infty$ and $\pi r^{2}=1$, we can write

$$
P\left(V_{k}>0\right)=p_{1}+p_{2}+p_{3},
$$

where

$$
\begin{aligned}
p_{1} & \equiv P(\text { no disk is centered within } R) \\
& =\exp \left(-\lambda \ell^{2}\right) \rightarrow 0,
\end{aligned}
$$

$$
\begin{aligned}
p_{2} \equiv & P(\text { at least one disk is centered within } R, \text { but } \\
& \text { none of the disks intersects any other disk and } \\
& \text { none of the disks intersect the boundary of } R) \\
\leq & P(\text { at least one disk is centered within } R) \\
& \times P(\text { a given disk intersects no other disks }) \\
= & \left(1-\exp \left(-\lambda \ell^{2}\right)\right) \times \exp \left(-\lambda \pi(2 r)^{2}\right) \\
\leq & \exp (-4 \lambda) \rightarrow 0,
\end{aligned}
$$

and

$$
\begin{aligned}
p_{3} \equiv & P(R \text { is not completely } k \text {-covered, at least } \\
& \text { one disk is centered within } R, \text { and at least } \\
& \text { two disks intersect each other or at least } \\
& \text { one disk intersects the boundary of } R) .
\end{aligned}
$$

Therefore

$$
P\left(V_{k}>0\right) \rightarrow p_{3} \text { as } \ell \rightarrow+\infty .
$$

Next we derive an upper bound of $p_{3}$.

If $R$ is not completed $k$-covered, if one or more disks are centered within $R$, and if there exist crossings in $R$, then at least one of the disks has two or more un- $k$-covered crossings on its boundary. Let $M_{k}$ denote the number of crossings that are not $k$-covered. Then we have

$$
p_{3} \leq P\left(M_{k} \geq 2\right) \leq E\left(M_{k}\right) / 2 .
$$

We first consider crossings created by two disks intersecting each other. The expected number, $D$, of nodes in region $R$ is $\lambda \ell^{2}$. If any two nodes are within a distance of $2 r$ from each other, their coverage disks intersect. Hence, the expected number of crossings created by a given node is $2 \lambda \pi(2 r)^{2}$. Since each crossing is counted twice, the expected value of the total number, $N_{1}$, of crossings created by two disks intersecting each other is given by

$$
E\left(N_{1}\right)=\lambda \ell^{2} \cdot \lambda \pi(2 r)^{2}=4 \lambda^{2} \ell^{2} .
$$

Now we consider crossings created by a disk intersecting the boundary of region $R$. If a node is within a distance of $r$ to the boundary of region $R$, at most two crossings will be created, except when the node is located on the corner of region $R$ (e.g., Region 3 in Fig. 1). In that case, at most 4 crossings will be created. Hence the expected value of the total number, $N_{2}$, of crossings created by a disk intersecting the boundary of region $R$ is given by

$$
E\left(N_{2}\right) \leq 8 \lambda \ell r .
$$

Recall that the probability that a given crossing is not $k$ covered is $e^{-\lambda} \sum_{i=0}^{k-1} \lambda^{i} / i$ ! (Eq. (2)). By Eqs. (11) and (12), we have

$$
\begin{aligned}
E\left(M_{k}\right) & =\left(E\left(N_{1}\right)+E\left(N_{2}\right)\right) \cdot e^{-\lambda} \sum_{i=0}^{k-1} \frac{\lambda^{i}}{i !} \\
& \leq\left(4 \lambda^{2} \ell^{2}+8 \lambda \ell r\right) e^{-\lambda} \sum_{i=0}^{k-1} \frac{\lambda^{i}}{i !} \\
& =4 \lambda^{2} \ell^{2}(1+o(1)) \cdot e^{-\lambda} \sum_{i=0}^{k-1} \frac{\lambda^{i}}{i !} .
\end{aligned}
$$


Since $\lambda \rightarrow+\infty$ as $\ell \rightarrow+\infty$, by Eqs. (10) and (13) we have

$$
\begin{aligned}
p_{3} & \leq 2 \lambda^{2} \ell^{2}(1+o(1)) e^{-\lambda} \sum_{i=0}^{k-1} \frac{\lambda^{i}}{i !} \\
& =2 \ell^{2} e^{-\lambda} \frac{\lambda^{k+1}}{(k-1) !}(1+o(1)) .
\end{aligned}
$$

If $\lambda=\log \ell^{2}+(k+1) \log \log \ell^{2}+c(\ell)$, then $\lambda_{1} \equiv \lambda-\log \ell^{2}=$ $(k+1) \log \log \ell^{2}+c(\ell)$. By the reasoning at the beginning of the proof, we can assume $c(\ell)=o\left(\log \ell^{2}\right)$ as $\ell \rightarrow+\infty$, and hence $\lambda_{1}=o\left(\log \ell^{2}\right)$. This gives $\lambda^{k+1}=\left(\log \ell^{2}\right)^{k+1}(1+o(1))$, and hence

$$
\begin{aligned}
p_{3} & \leq \frac{2 \lambda^{k+1}}{\left(\log \ell^{2}\right)^{k+1} e^{c(\ell)}(k-1) !}(1+o(1)) \\
& =\frac{2(1+o(1))}{e^{c(\ell)}(k-1) !} .
\end{aligned}
$$

Since $c(\ell) \rightarrow+\infty$ as $\ell \rightarrow+\infty, p_{3} \rightarrow 0$ as $\ell \rightarrow+\infty$. The first part is proved.

Now we prove that if $c(\ell) \leq C$ for some finite $C$ as $\ell \rightarrow$ $+\infty, P\left(V_{k}>0\right) \geq \epsilon$ for $\epsilon=1 /\left(1+4 e^{C}(k+1) !\right)$. Let $I$ be the indicator function, by the Cauchy-Schwartz inequality, we have

$$
\begin{aligned}
E\left(V_{k}\right) & =E\left(V_{k} I\left(V_{k}>0\right)\right) \\
& \leq\left(E\left(V_{k}^{2}\right) E\left(I^{2}\left(V_{k}>0\right)\right)\right)^{1 / 2} \\
& =\left(E\left(V_{k}^{2}\right) P\left(V_{k}>0\right)\right)^{1 / 2},
\end{aligned}
$$

and

$$
P\left(V_{k}>0\right) \geq \frac{\left(E V_{k}\right)^{2}}{E\left(V_{k}^{2}\right)} .
$$

Eq. (4) gives the expression of $E\left(V_{k}\right)$. We now derive the bound of $E\left(V_{k}^{2}\right)$. By definition, we have

$$
\begin{aligned}
E\left(V_{k}^{2}\right) & =E\left(\iint_{R^{2}} \chi_{k}\left(x_{1}\right) \chi_{k}\left(x_{2}\right) d x_{1} d x_{2}\right) \\
& =\iint_{R^{2}} E\left(\chi_{k}\left(x_{1}\right) \chi_{k}\left(x_{2}\right)\right) d x_{1} d x_{2} \\
& \equiv I_{1}+I_{2},
\end{aligned}
$$

where

$$
\begin{aligned}
I_{1} & \equiv \iint_{R^{2} \cap\left\{\left|x_{1}-x_{2}\right|>2 r\right\}} E\left(\chi_{k}\left(x_{1}\right) \chi_{k}\left(x_{2}\right)\right) d x_{1} d x_{2} \\
I_{2} & \equiv \iint_{R^{2} \cap\left\{\left|x_{1}-x_{2}\right| \leq 2 r\right\}} E\left(\chi_{k}\left(x_{1}\right) \chi_{k}\left(x_{2}\right)\right) d x_{1} d x_{2} .
\end{aligned}
$$

For $\left|x_{1}-x_{2}\right|>2 r, \chi_{k}\left(x_{1}\right)$ and $\chi_{k}\left(x_{2}\right)$ are independent, and $E\left(\chi_{k}(x)\right)=e^{-\lambda} \sum_{i=0}^{k-1} \lambda^{i} / i$ ! for all $x$. Hence,

$$
\begin{aligned}
I_{1} & \equiv \iint_{R^{2} \cap\left\{\left|x_{1}-x_{2}\right|>2 r\right\}} E\left(\chi_{k}\left(x_{1}\right) \chi_{k}\left(x_{2}\right)\right) d x_{1} d x_{2} \\
& =\iint_{R_{k}}\left(x_{1}\right) E \chi_{k}\left(x_{2}\right) d x_{1} d x_{2} \\
& \leq \iint_{R^{2}} E \chi_{k}\left(x_{1}\right) E \chi_{k}\left(x_{2}\right) d x_{1} d x_{2} \\
& =\left(\ell^{2} e^{-\lambda} \sum_{i=0}^{k-1} \frac{\lambda^{i}}{i !}\right)^{2}=\left(E\left(V_{k}\right)\right)^{2}
\end{aligned}
$$

What is left is the derivation of $I_{2}$. Let $B_{1}$ and $B_{2}$ denote the unit-area disks centered at $x_{1}$ and $x_{2}$, respectively. If $\left|x_{1}-x_{2}\right|=x \leq 2 r$ and $x_{1}$ and $x_{2}$ are given, then

$$
\begin{aligned}
& E\left(\chi_{k}\left(x_{1}\right) \chi_{k}\left(x_{2}\right)\right) \\
&= P\left(\text { Both } B_{1} \text { and } B_{2} \text { contain less than } k \text { nodes }\right) \\
& \leq P\left(B_{1} \text { contains less than } k \text { nodes, } B_{2}-B_{1}\right. \\
&\text { contains less than } k \text { nodes }) \\
&= P\left(B_{1} \text { contains less than } k \text { nodes }\right) \times \\
& P\left(B_{2}-B_{1} \text { contains less than } k \text { nodes }\right)
\end{aligned}
$$

The last equality results from the fact that $B_{1}$ and $B_{2}-B_{1}$ are disjoint and thus the number of nodes that are located in them are independent (under the Poisson point process assumption).

$$
P\left(B_{1} \text { contains less than } k \text { nodes }\right)=e^{-\lambda} \sum_{i=0}^{k-1} \frac{\lambda^{i}}{i !} .
$$

Let $B(u)$ denote the intersection area of the two unit-area disks whose centers are $2 u$ apart. Then,

$$
B(u)=4 \int_{u}^{1}\left(1-y^{2}\right)^{1 / 2} d y=\pi-4 \int_{0}^{u}\left(1-y^{2}\right)^{1 / 2} d y
$$

Now the second term of Eq. (22) can be expressed as

$$
\begin{aligned}
\int_{0}^{u}\left(1-y^{2}\right)^{1 / 2} d y & =(u / 2)\left\{u^{-1} \arcsin u+\left(1-u^{2}\right)^{1 / 2}\right\} \\
& \geq(u / 2) \arcsin 1=(\pi / 4) u
\end{aligned}
$$

since $u^{-1} \arcsin u+\left(1-u^{2}\right)^{1 / 2}$ is decreasing on $(0,1)$. Hence the area of $B_{2}-B_{1}$ is

$$
\begin{aligned}
\left\|B_{2}-B_{1}\right\| & =r^{2}(\pi-B(x /(2 r))) \\
& \geq r^{2} \cdot 4(\pi / 4) \cdot x /(2 r)=x /(2 r) .
\end{aligned}
$$

Therefore,

$$
\begin{aligned}
P & \left(B_{2}-B_{1} \text { contains less than } k \text { nodes }\right) \\
& =e^{-\lambda\left\|B_{2}-B_{1}\right\|} \sum_{i=0}^{k-1} \frac{\left(\lambda\left\|B_{2}-B_{1}\right\|\right)^{i}}{i !} \\
& \leq e^{-\lambda x /(2 r)} \sum_{i=0}^{k-1} \frac{(\lambda x /(2 r))^{i}}{i !},
\end{aligned}
$$

since $e^{-x} \sum_{i=0}^{k-1} x^{i} / i$ ! is decreasing on $[0,+\infty)$.

By Eqs. (20), (21) and (24), we can express $I_{2}$ as

$$
\begin{aligned}
I_{2} \equiv & \iint_{R^{2} \cap\left\{\left|x_{1}-x_{2}\right| \leq 2 r\right\}} E\left\{\chi_{k}\left(x_{1}\right) \chi_{k}\left(x_{2}\right)\right\} d x_{1} d x_{2} \\
\leq & \int_{R} d x_{1} \int_{0}^{2 r}\left(e^{-\lambda} \sum_{i=0}^{k-1} \frac{\lambda^{i}}{i !}\right) \\
& \cdot\left(e^{-\lambda x /(2 r)} \sum_{i=0}^{k-1} \frac{(\lambda x /(2 r))^{i}}{i !}\right) 2 \pi x d x \\
= & \ell^{2}\left(e^{-\lambda} \sum_{i=0}^{k-1} \frac{\lambda^{i}}{i !}\right)\left(\int_{0}^{1} e^{-\lambda u} \sum_{i=0}^{k-1} \frac{(\lambda u)^{i}}{i !} 8 u d u\right),
\end{aligned}
$$

where the last equality is obtained by changing variable $u=$ $x /(2 r)$. The third factor in Eq. (25) can be further simplified as follows.

$$
\begin{aligned}
& \int_{0}^{1} e^{-\lambda u} \sum_{i=0}^{k-1} \frac{(\lambda u)^{i}}{i !} \cdot 8 u d u \\
& \quad \leq \int_{0}^{+\infty} e^{-\lambda u} \sum_{i=0}^{k-1} \frac{(\lambda u)^{i}}{i !} \cdot 8 u d u
\end{aligned}
$$




$$
\begin{aligned}
& =\int_{0}^{+\infty} e^{-\lambda u} \sum_{i=0}^{k-1} \frac{\lambda^{i} u^{i+1}}{i !} \cdot 8 d u \\
& =\sum_{i=0}^{k-1} \frac{\lambda^{-2} \Gamma(i+2)}{i !} \cdot 8 \\
& =\lambda^{-2} \sum_{i=0}^{k-1}(i+1) \cdot 8 \\
& =4 k(k+1) \lambda^{-2} .
\end{aligned}
$$

Hence we have

$$
I_{2} \leq 4 k(k+1) \lambda^{-2} \ell^{2}\left(e^{-\lambda} \sum_{i=0}^{k-1} \frac{\lambda^{i}}{i !}\right) .
$$

Combining Eqs. (17), (18), (19) and (27), we have

$$
\begin{aligned}
P & \left(V_{k}>0\right) \\
& =\frac{E\left(V_{k}\right)^{2}}{E\left(V_{k}^{2}\right)} \\
& \geq \frac{\left(E V_{k}\right)^{2}}{\left(E V_{k}\right)^{2}+4 k(k+1) \lambda^{-2} \ell^{2}\left(e^{-\lambda} \sum_{i=0}^{k-1} \frac{\lambda^{i}}{i !}\right)} \\
& \equiv \frac{1}{1+\beta},
\end{aligned}
$$

where

$$
\begin{aligned}
\beta & \equiv \frac{4 k(k+1) \lambda^{-2} \ell^{2}\left(e^{-\lambda} \sum_{i=0}^{k-1} \frac{\lambda^{i}}{i !}\right)}{\left(E V_{k}\right)^{2}} \\
& =\frac{4 k(k+1) \lambda^{-2} \ell^{2}\left(e^{-\lambda} \sum_{i=0}^{k-1} \frac{\lambda^{i}}{i !}\right)}{\left(\ell^{2} e^{-\lambda} \sum_{i=0}^{k-1} \frac{\lambda^{i}}{i !}\right)^{2}} \\
& =\frac{4 k(k+1) \lambda^{-2}}{\ell^{2} e^{-\lambda} \sum_{i=0}^{k-1} \frac{\lambda^{i}}{i !}} \\
& \leq \frac{4 k(k+1)}{\ell^{2} e^{-\lambda} \lambda^{k+1} /(k-1) !} .
\end{aligned}
$$

Let $\lambda_{1} \equiv \lambda-\log \ell^{2}=(k+1) \log \log \ell^{2}+c(\ell)$. By the assumption $c(\ell) \leq C$, with sufficiently large $\ell$, we have $\lambda_{1}>0$, and

$$
\beta \leq \frac{4 e^{c(\ell)}\left(\log \ell^{2}\right)^{k+1}(k+1) !}{\left(\log \ell^{2}+\lambda_{1}\right)^{k+1}} \leq 4 e^{C}(k+1) ! .
$$

It then follows from Eqs. (28) and (29) that

$$
P\left(V_{k}>0\right) \geq \frac{\left(E V_{k}\right)^{2}}{E\left(V_{k}^{2}\right)} \geq \frac{1}{1+4 e^{C}(k+1) !} .
$$

This completes the proof.

Remark 1. If we let $c(\ell) \rightarrow-\infty$, and $\lambda=\log \ell^{2}+(k+$ 1) $\log \log \ell^{2}+c(\ell)$, we can conclude $P\left(V_{k}>0\right) \rightarrow 1$ as $\ell \rightarrow$ $+\infty$.

Remark 2. If we let $-(k+1) \log \log \ell^{2} \leq c(\ell) \leq C$, then $P\left(V_{k}>0\right) \geq 1 /\left(1+4 e^{C}(k+1) !\right)$ is true for any finite $\ell$, since the second part of the proof does not require any asymptotic property in this case.

Remark 3. The terms "complete $k$-coverage" and " $V_{k}=$ 0 " have been used interchangeably, as it has been proved (for the case of $k=1$ ) in [11] that the probability of their difference goes to 0 if the region is open and the coverage shape (e.g., the disk in the paper) is closed. It has also been stated in [11] that the same conclusion holds for any regular region and shape. (The interested reader is referred to the discussions following Theorem 3.3 in [11]). The proof can also be extended to the case of any finite $k$.

The following corollary is an obvious consequence of Theorem 1 and Remark 1.

Corollary 1. If $\lambda=\log \ell^{2}+(k+2) \log \log \ell^{2}+c(\ell)$, and $c(\ell) \rightarrow-\infty$ as $\ell \rightarrow+\infty$, then the upper bound of the 1-lifetime is $k T$ with probability approaching 1 , where $T$ is the lifetime of each sensor.

It is interesting to observe from Corollary 1 that the node density required to achieve a 1-lifetime of $k T$ is not equal to $k$ times the required density for asymptotic coverage. As a matter of fact, the former is much smaller than the latter. This is because with a larger node density, one can make better use of coverage areas of sensor nodes. This trend will be confirmed again in the following sections.

\section{UPPER BOUND OF $\alpha$-LIFETIME IN FINITE REGIONS}

The asymptotic upper bound of the 1-lifetime derived in Section 4 gives the required node density in order to achieve complete coverage as the monitored area grows to infinity $(\ell \rightarrow \infty)$. However in practice one may be more interested in knowing how many nodes should be deployed (or, equivalently, what is the node density) in order to achieve the $\alpha$-lifetime in a finite region. Results derived in Section 4 cannot be directly applied to answer this question, as they are derived for complete coverage for infinitely large regions.

In this section, we consider the $\alpha$-lifetime in a finite region with a finite density of sensor nodes, where $0<\alpha<1$ and usually $\alpha$ is close to 1 . We derive two bounds: (i) an upper bound of $\alpha$-lifetime for a special family of algorithms in which the entire region is completely covered initially, and the coverage ratio is gradually reduced until it drops below a certain threshold $\alpha$; and (ii) an upper bound of $\alpha$-lifetime that applies to algorithms that maintain the coverage ratio of $\alpha$ from the beginning of network deployment. The second bound applies to any algorithm.

\subsection{Upper Bound of $\alpha$-Lifetime for a Special Family of Algorithms}

We first derive the upper bound of $\alpha$-lifetime for the family of algorithms that intend to completely cover the region initially and gradually reduce the coverage ratio, until it drops below a certain threshold $\alpha$.

We can divide the entire region $R$ into several sub-regions $R_{0}, R_{1}, \cdots, R_{n}$, where all points in $R_{i}$ are exactly covered by $i$ sensor nodes (Fig. 2). Thus $V_{k}=\sum_{i=0}^{k-1}\left\|R_{i}\right\|$ and $1-V_{k} / \ell^{2}$ is the portion of the region in which each point is covered by at least $k$ nodes. We can also divide the network lifetime into rounds with the duration of each round set to $T$. In each round, a minimum set of nodes which are not chosen in previous rounds and have maximum coverage is chosen to operate. Thus after $k$ rounds, the maximum possible coverage ratio is at most $1-V_{k+1} / \ell^{2}$. Clearly, if $\alpha>$ $1-V_{k+1} / \ell^{2}$, the sensor network can not provide coverage ratio $\alpha$ any more. Thus the upper bound of $\alpha$-lifetime is

$$
L(\lambda, \alpha)=\max \left\{k: \alpha \leq 1-V_{k} / \ell^{2}\right\} \cdot T .
$$




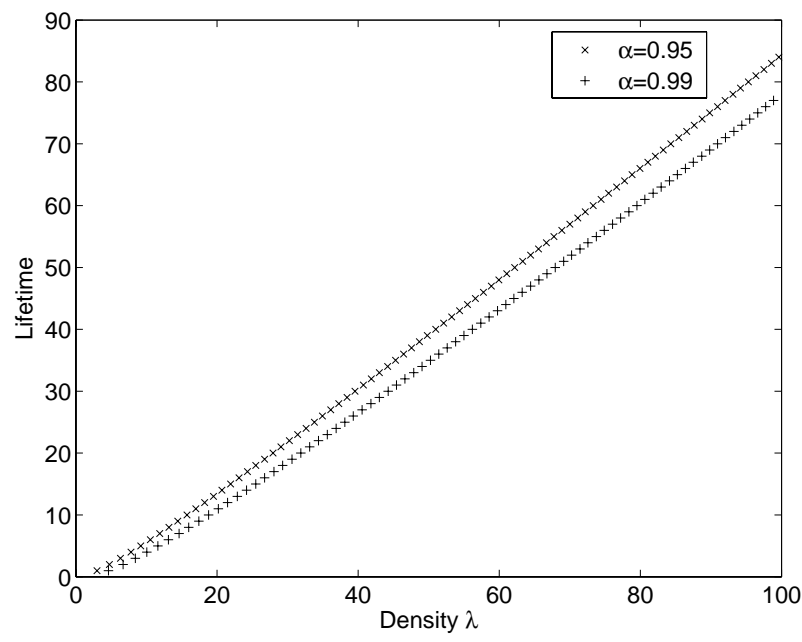

(a) Average $\alpha$-lifetime v.s. density

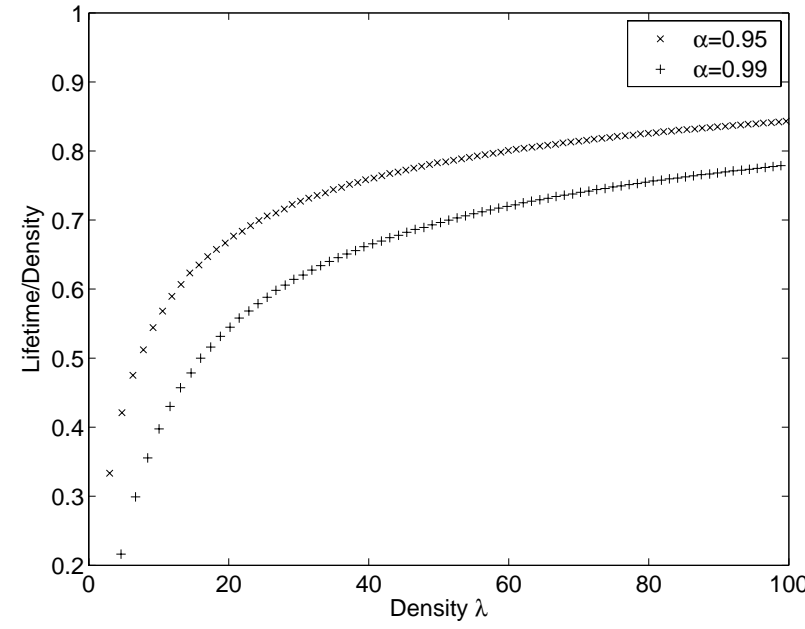

(b) Average $\alpha$-lifetime per unit of density v.s. density

Figure 3: Average $\alpha$-lifetime and $\alpha$-lifetime per unit of density versus density.

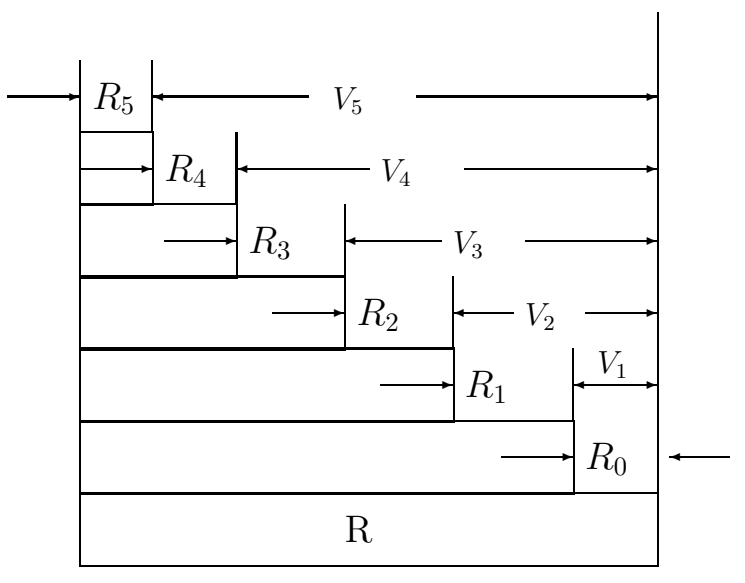

Figure 2: The entire region $R$ can be divided into different sub-regions: $R_{0}, R_{1}, \cdots, R_{n}$, where all points in $R_{i}$ are exactly covered by $i$ nodes.

As $V_{k}$ 's are random variables whose distributions are difficult, if not impossible, to obtain. For analysis tractability, we use $E\left(V_{k}\right)$ to approximate $V_{k}$ and hence the resulting $\alpha$-lifetime can be regarded as the average $\alpha$-lifetime:

$$
G(\lambda, \alpha)=\max \{k: \alpha \leq 1-F(k, \lambda)\} \cdot T,
$$

where

$$
F(k, \lambda)=E\left(V_{k}\right) / \ell^{2}=\exp (-\lambda)\left(\sum_{i=0}^{k-1} \frac{\lambda^{i}}{i !}\right) .
$$

As a matter of fact, $G(\lambda, \alpha)$ is not the expectation of $L(\lambda, \alpha)$. However, we prove in the following theorem that it suffices to approximate $V_{k}$ with $E\left(V_{k}\right)$ in regions of large area.

Theorem 2. As $\ell \rightarrow+\infty$ and $n / \ell^{2} \rightarrow \lambda, V_{k} / \ell^{2} \rightarrow F(k, \lambda)$ almost surely, where $F(k, \lambda)$ is defined in Eq. (33).

The proof is omitted due to space limit. Please refer to [24] for the proof.

Numerical examples. Figure 3 depicts the average $\alpha$ lifetime $G(\lambda, \alpha)$ and the average $\alpha$-lifetime per unit density $G(\lambda, \alpha) / \lambda$ versus the minimum density $\lambda$ required to achieve the average $\alpha$-lifetime of $k T$ under the cases of $\alpha=0.95$ and 0.99 , where we let $T=1$. The average $\alpha$-lifetime per unit density increases as the node density $\lambda$ increases. This coincides with our earlier observation in the asymptotic case that a higher density of sensors leads to better utilization of coverage areas.

\subsection{Upper Bound of $\alpha$-Lifetime for All Algorithms}

Several sensor network applications do not require that full coverage be maintained. Instead it is sufficient to maintain the coverage ratio above a certain threshold $\alpha$ throughout the network lifetime. In this case, energy can be saved by maintaining $\alpha$-coverage since the network is initialized. In this section, we derive the upper bound of the network lifetime in this case. Note that this upper bound can be applied to all algorithms that maintain $\alpha$-coverage. For analysis tractability, again we use $E\left(V_{k}\right)$ to approximate $V_{k}$. The following theorem establishes the upper bound of the lifetime.

ThEOREM 3. Let $\gamma_{i} \triangleq 1-E\left(V_{i}\right) / l^{2}$ and $\beta_{i} \triangleq \gamma_{i}-\gamma_{i+1}$. Then the upper bound of $\alpha$-lifetime for a sensor network with density $\lambda$ is

$$
\left\lfloor\min _{k: \alpha>\gamma_{k}} H(k, \alpha) \triangleq \frac{\sum_{i=1}^{k-1} i \beta_{i}}{\alpha-\gamma_{k}}\right\rfloor \cdot T,
$$

where $\lfloor x\rfloor$ is the maximum integer that is less than or equal to $x$. 


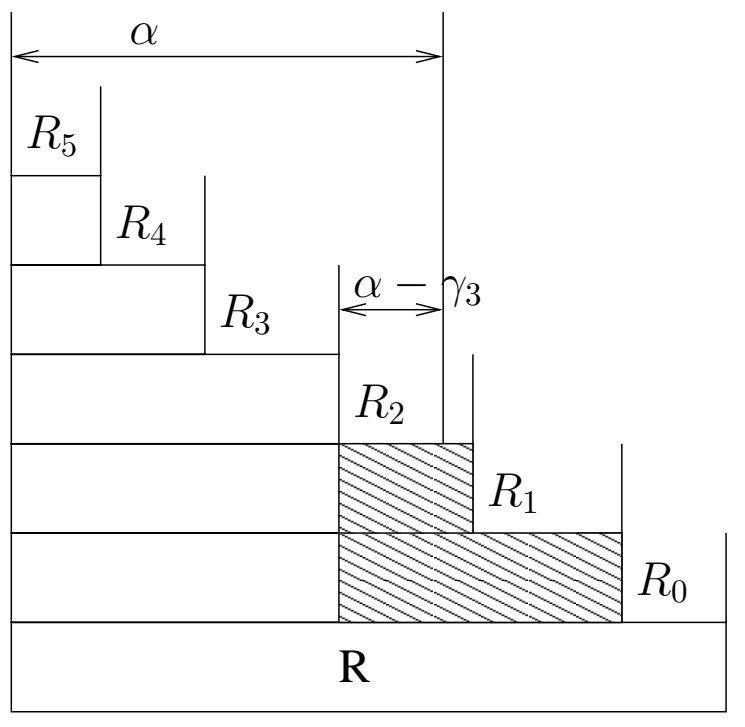

Figure 4: In each round, $\alpha-\gamma_{3}$ portion of the region must come from region $R_{2}$ and $R_{1}$ to ensure $\alpha$ coverage. The total lifetime "contribution" $R_{1}$ and $R_{2}$ can make over all rounds is $\beta_{1}+2 \beta_{2}$. Hence the $\alpha$-lifetime is upper bounded by $\left(\beta_{1}+2 \beta_{2}\right) /\left(\alpha-\gamma_{3}\right)$.

Proof. We still divide the entire region $R$ into different subregions $R_{0}, R_{1}, \cdots$, where all points in $R_{i}$ are exactly covered by $i$ nodes. By definition, $\gamma_{k}$ represents the portion of region $R$ that is covered by at least $k$ nodes and $\beta_{k}$ represents the portion of region $R$ that is covered by exactly $k$ nodes. Thus $\beta_{k}=\left\|R_{k}\right\| /\|R\|$.

For each $k$ such that $\gamma_{k}<\alpha$, in each round of time $T$, the working nodes must cover $\alpha$ portion of the region $R$, among which at least $\alpha-\gamma_{k}$ portion must come from $R_{1} \cup \cdots \cup R_{k-1}$ since $\cup_{i \geq k} R_{i}$ can provide at most $\gamma_{k}$ coverage (and $R_{0}$ is not covered by any node). On the other hand, for each $i<k$, the total coverage contribution of region $R_{i}$ throughout the lifetime is at most $i \beta_{i}$ (since it can provide $\beta_{i}$ portion of coverage for $i$ rounds). Hence, the total amount of coverage $R_{1}, R_{2}, \cdots, R_{k-1}$ can contribute throughout the lifetime over all rounds is $\sum_{i=1}^{k-1} i \beta_{i}$. Therefore, the maximum lifetime is upper bounded by

$$
\left\lfloor\frac{\sum_{i=1}^{k-1} i \beta_{i}}{\alpha-\gamma_{k}}\right\rfloor \cdot T .
$$

Since this is true for every $k$ such that $\alpha>\gamma_{k}$, the $\alpha$-lifetime is upper bounded by Eq. (34).

As an example, as shown in Figure 4, in each round, $\alpha-\gamma_{3}$ portion of the region must come from region $R_{2}$ and $R_{1}$ to ensure $\alpha$-coverage since $\alpha>\gamma_{3}$. The total lifetime "contribution" $R_{1}$ and $R_{2}$ can make over all rounds is $\beta_{1}+2 \beta_{2}$. Hence the $\alpha$-lifetime is upper bounded by $\left(\beta_{1}+2 \beta_{2}\right) /\left(\alpha-\gamma_{3}\right)$.

Recall in the proof of Theorem 3 , in each round we divide the entire region into two sub-regions. In the first subregion, each point is covered by at least $k$ nodes and in the second sub-region, each point is covered by at most $k-1$ nodes. The proof of Theorem 3 only considers the limit implied by the second sub-region. In what follows, we prove that the first sub-region can always provide $\gamma_{k}$ portion coverage for at least $\lfloor H(k, \alpha)\rfloor$ rounds for the $k$ that minimizes
$H(k, \alpha)$.

TheOREM 4. Let $k=\arg \min _{i: \alpha>\gamma_{i}} H(i, \alpha)$, then

$$
k \geq H(k, \alpha) \text {. }
$$

Proof. To facilitate the proof, we first give several nice properties of $H(k, \alpha)$ in the next lemma.

Lemma 1. For all $k$ such that $\alpha>\gamma_{k}, H(k, \alpha)$ given in Eq. (34) has the following properties:

(i) If $H(k, \alpha)>k, H(k, \alpha)$ monotonically decreases as $k$ increases;

(ii) If $H(k, \alpha)<k, H(k, \alpha)$ monotonically increases as $k$ increases;

(iii) If $H(k, \alpha)=k$, then $H(k, \alpha)=H(k+1, \alpha)$;

(iv) If $H(k, \alpha)>k$, then $H(k+1, \alpha)>k$;

(v) If $H(k, \alpha)=k$, then $H(k+1, \alpha)=k$;

(vi) If $H(k, \alpha)<k$, then $H(k+1, \alpha)<k$.

Proof. Refer to Appendix B.

Now since $k=\arg \min _{i: \alpha>\gamma_{i}} H(i, \alpha)$, if $H(k, \alpha)>k$, by property (i) in Lemma $1, H(k, \alpha)>H(k+1, \alpha)$. Since $\gamma_{k+1}<\gamma_{k}<\alpha$, this contradicts our assumption that $k=$ $\arg \min _{i: \alpha>\gamma_{i}} H(i, \alpha)$. So $H(k, \alpha) \leq k$.

The above theorem can be used to prove that the lifetime upper bound given in Theorem 3 is tight. This is given in the next Corollary.

Corollary 2. The upper bound in Theorem 3 is tight if we can arbitrarily select the region(s) to be covered to provide exactly $\alpha$-coverage in each round.

Proof. In each round, we select all the regions that are covered by at least $k$ nodes (which is $\gamma_{k}$ portion of the region $R$ ). In addition, we select $\alpha-\gamma_{k}$ portion in the regions that are covered by less than $k$ nodes. Then in each round $\alpha$ coverage is achieved and at least $\lfloor H(k, \alpha)\rfloor$ rounds can be supported because $H(k, \alpha) \leq k$.

Numerical examples. Figure 5 gives the upper bound of the lifetime derived in Section 5.1 and that in this subsection, and their respective lifetime per unit of density. As compared with the upper bound of the lifetime derived in Section 5.1, the "universal" upper bound of the lifetime increases by $15 \%$ for $99 \%$-coverage and over $20 \%$ for $95 \%$ coverage. The upper bound of the lifetime per unit density increases as the density increases in general, and slightly decreases at certain density values. This is because the upper bound of the lifetime does not change for the slight increase in the node density $\lambda$. It is not surprising to observe that the lifetime per unit density can be more than 1 in some cases, because less than 100\%-coverage is required in each round.

Another interesting finding is that although it is, in general, desirable to deploy sensors with high density to achieve a large lifetime per unit of nodal density, the increase in the lifetime per unit of nodal density levels off when the density exceeds certain threshold. The overhead incurred in maintaining coverage in a distributed manner dominates when the sensor density becomes very high. 


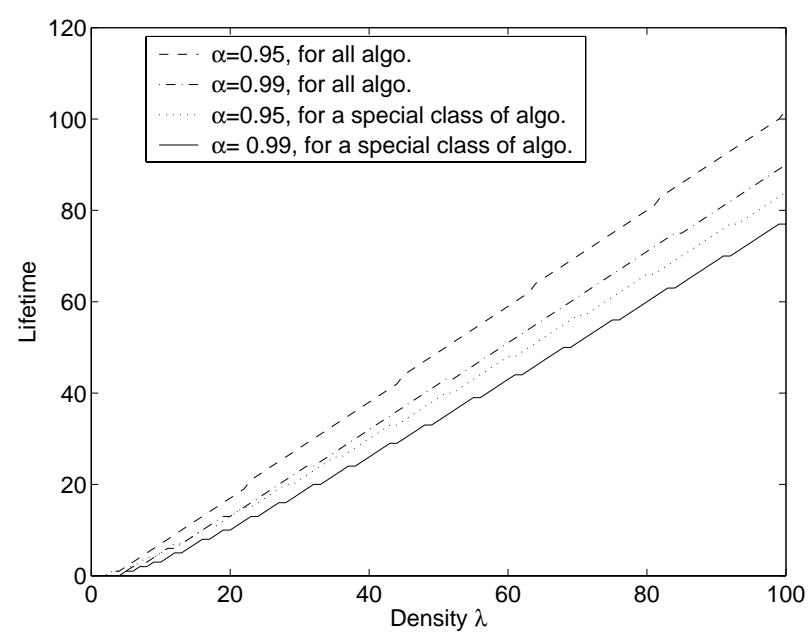

(a) Average $\alpha$-lifetime v.s. density

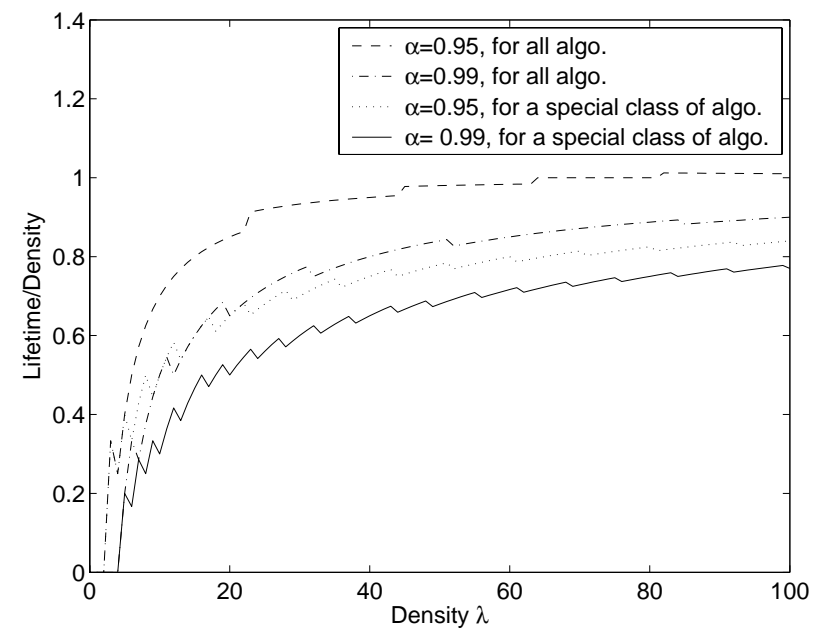

(b) Average $\alpha$-lifetime per unit of density v.s. density

Figure 5: Average $\alpha$-lifetime and $\alpha$-lifetime per unit of density derived in Section 5.1 and in Section 5.2.

\section{SIMULATION STUDY}

In this section, we carry out several sets of simulations to validate the theoretical lifetime upper bound. Since it is difficult, if not impossible, to simulate 1-lifetime for the infinite dimension case, our simulation study focuses on validating the upper bound of $\alpha$-lifetime in a reasonably large area.

\subsection{Simulation Methodology}

We use $N$ independently and randomly distributed disks with uniform distribution to approximate a Poisson point process. In a square region with $1000 \times 1000$ pixels, we randomly generate $N$ disks, each with radius $r$. The centers of the $N$ disks are independently and randomly distributed in the square region with uniform distribution. Then for each pixel we count the number of disks that cover it. We term this as the coverage number of each pixel. For each value of $\alpha$, we calculate $V_{i}$ as the number of pixels that have coverage number less than $i$. With the simulated value of $V_{i}$, we calculate the upper bound of the lifetime for the special class of algorithms using Eq. (31), and that for all algorithms using Eq. (34). These upper bounds thus calculated correspond to the theoretical upper bounds of the lifetime in Section 5.1 and in Section 5.2 respectively. The lifetime of a single sensor $T$ is set to 1 . The network density is evaluated as

$$
\frac{N \pi r^{2}}{1000000}
$$

All the results reported below are averages of 50 simulation runs.

Note that decreasing the disk radius with the side length of the square area fixed has the same effect of increasing the side length of the square area with the disk radius fixed. For each value of $\alpha$ we vary the disk radii over different runs (but keep the radii of all disks constant in each run) to investigate how the area size of the region affects the upper bound of the lifetime. For each value of $\alpha$ and disk radius, we vary the number of sensors to change the node density. As the same trend has been observed for different values of $\alpha$, in what follows we only report results for $\alpha=0.95$.

\subsection{Simulation Results}

Figure 6 shows the theoretical and simulated upper bound of the network lifetime. In particular, Fig. 6 (a) shows the upper bound of the lifetime for a special class of algorithms, derived in Section 5.1, while Fig. 6 (b) shows the upper bound of the lifetime for all algorithms, derived in Section 5.2. When the disk radius decreases (which is equivalent to increasing the side length of the area but keeping the disk radius fixed), the simulated upper bounds become closer to the theoretical upper bounds. This is exactly what Theorem 2 states: when the size of the monitored region increases, $V_{k}$ will asymptotically converge to $E\left(V_{k}\right)$, and thus we can use $E\left(V_{k}\right)$ to approximate $V_{k}$ for large sensor networks. In addition, Fig. 6 also suggests that even when the monitored region is small, the theoretical upper bound is still an upper bound, although it may not be tight.

\section{CONCLUSIONS}

In this paper we have investigated the upper bound of $\alpha$ lifetime for large scale sensor networks. We first derive the asymptotic node density required to ensure full coverage for the duration of $k$ times the lifetime of a single sensor (in the almost surely sense) in large sensor networks, as the network size approaches infinity. Then we derive the upper bound of $\alpha$-lifetime in a finite region with a finite density of nodes. In particular, we derive two bounds: (i) an upper bound of $\alpha$ lifetime for a special family of algorithms in which the entire region is completely covered initially, and the coverage ratio is gradually reduced until it drops below a certain threshold $\alpha$; and (ii) an upper bound of $\alpha$-lifetime that applies to algorithms that maintain the coverage ratio of $\alpha$ from the beginning of network deployment. We carry out several sets of simulations to validate the derived results. The simulation results indicate that the derived upper bounds of the 


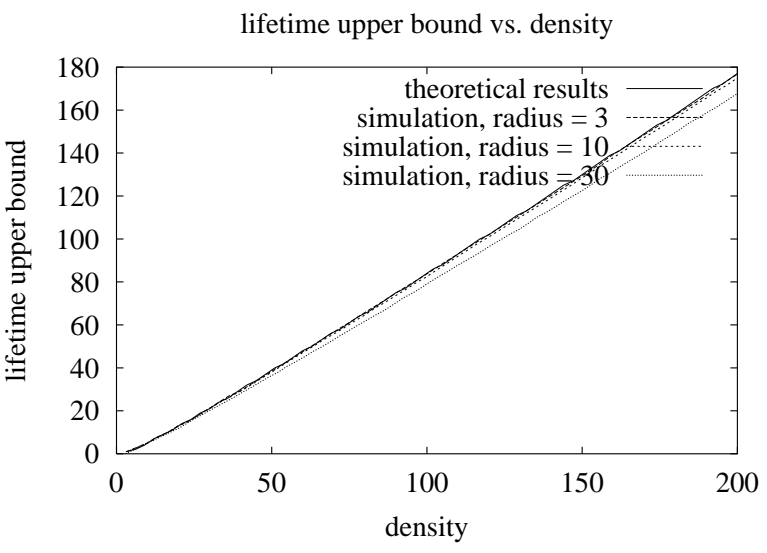

(a) Average 95\%-lifetime derived for a special class of algorithms

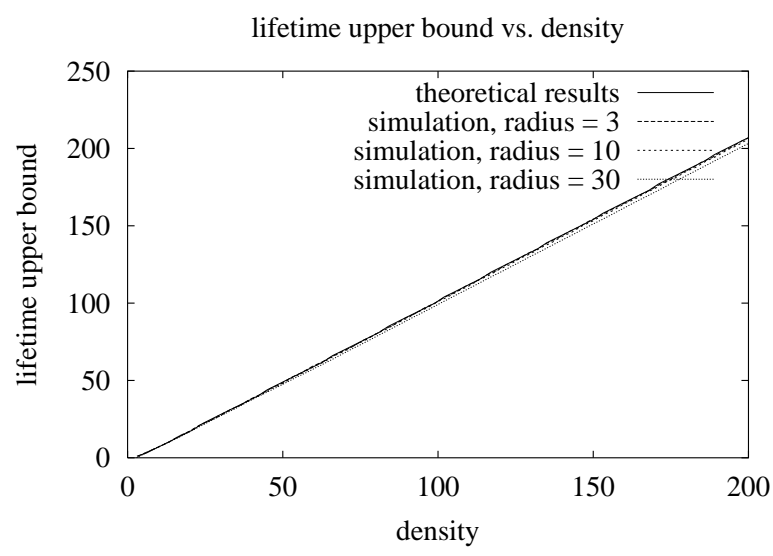

(b) Average 95\%-lifetime derived for all algorithms

Figure 6: Average 95\%-lifetime derived in Section 5.1 and in Section 5.2 respectively.

network lifetime apply not only to extremely large areas, but also to small areas, although the derived upper bounds for the latter case may not be tight.

With our derivation, we are able to determine, given the lifetime $T$ of a single sensor node, how many sensor nodes have to be deployed in a region, in order to continuously monitor the region for a period of $k \cdot T$. Also, we observe that although it is, in general, desirable to deploy sensors with high density to achieve a large lifetime per unit of nodal density, the increase in the lifetime per unit of nodal density becomes marginal when the density exceeds certain threshold. The overhead incurred in maintaining coverage in a distributed manner dominates when the sensor density becomes high.

We have identified several research avenues. In particular, we will relax the assumption that the radio transmission range is at least twice as large as the sensing range, and derive the upper bound of the network lifetime by considering jointly coverage and connectivity. Note, however, that the upper bounds derived in this paper serve as upper bounds (although not as tight) even when the assumption does not hold.

\section{ACKNOWLEDGEMENT}

Thanks Will Chen and Rong Zheng for providing measurement results on sensing range of acoustic sensor on Berkeley Motes.

\section{REFERENCES}

[1] I. F. Akyildiz, W. Su, Y. Sankarasubramaniam, and E. Cayirci. Wireless Sensor Networks: A Survey, Computer Networks. March 2002.

[2] KEYENCE America. http://www.keyence.com/products/sensors.html.

[3] M. Bhardwaj and A. P. Chandrakasan. Bounding the lifetime of sensor network via optimal role assignments. In Proc. of IEEE Infocom 2002.
[4] M. Bhardwaj and A. P. Chandrakasan. Upper bounds on the lifetime of wireless sensor networks. In Proc. of IEEE International Conference on Communications (ICC) 01.

[5] Douglas M. Blough and P. Santi. Investigating upper bounds on network lifetime extension for cell-based energy conservation techniques in stationary ad hoc networks. In Proc. of ACM Mobicom 2002.

[6] B. Chen, K. Jamieson, H. Balakrishnan, and R. MOrris. Span: An energy-efficient operation in multihop wireless ad hoc networks. In Proc. of ACM MobiCom'01, 2001.

[7] S. Coleri, M. Ergen, and T. J. Koo. Lifetime analysis of a sensor network with hybrid automata modelling. In First ACM International Workshop on Wireless Sensor Network and Applications (WSNA02), Sep. 2002.

[8] D. Estrin, R. Govindan, J. S. Heidemann, and S. Kumar. Next century challenges: Scalable coordination in sensor networks. In Proc. of $A C M$ MobiCom'99, Washington, August 1999.

[9] L. Feeney and M. Nilsson. Investigating the energy consumption of a wireless network interface in an ad hoc networking environment. In Proc. of IEEE Infocom 2001.

[10] H. Gupta, S. Das, and Q. Gu. Connected sensor cover: Self-organization of sensor networks for efficient query execution. In Proc. of Mobihoc 2003.

[11] P. Hall. Introduction to the Theory of Coverage Processes. John Wiley and Sons, 1988.

[12] Crossbow Technology Inc. http://www.xbow.com/support/support_pdf_files/mtsmda_series_user_manual_revb.pdf.

[13] J. M. Kahn, R. H. Katz, and K. S. J. Pister. Next century challenges: Mobile networking for "smart dust". In Proc. of ACM MobiCom'99, August 1999.

[14] A. Mainwaring, J. Polastre, R. Szewczyk, and 
D. Culler. Wireless sensor networks for habitat monitoring. In First ACM International Workshop on Wireless Workshop in Wireless Sensor Networks and Applications (WSNA 2002), August 2002.

[15] M.D. Penrose. The longest edge of the random minimal spanning tree. Annals of Applied Probability, 7:340-361, 1997.

[16] S. G. Samko, A. A. Kilbas, and O. I. Marichev. Fractional Integrals and Derivatives, page 9. Yverdon, Switzerland: Gordon and Breach, 1993.

[17] Infrared Sensor. http://www.interq.or.jp/japan/se-inoue/e_pyro.htm.

[18] E. Shih, S. Cho, N. Ickes, R. Min, A. Sinha, A. Wang, and A. Chandrakasan. Physical layer driven protocol and algorithm design for energy-efficient wireless sensor networks. In Proc. of ACM MobiCom'01, Rome, Italy, July 2001.

[19] D. Tian and N. D. Georganas. A coverage-preserving node scheduling scheme for large wireless sensor networks. In First ACM International Workshop on Wireless Sensor Networks and Applications, Georgia, GA, 2002.

[20] X. Wang, G. Xing, Y. Zhang, C. Lu, R. Pless, and C. Gill. Integrated coverage and connectivity configuration in wireless sensor networks. In $A C M$ Sensys'03, Nov. 2003.

[21] Y. Xu, J. Heidemann, and D. Estrin. Geography-informed energy conservation for ad hoc routing. In Proc. of ACM MOBICOM'01, Rome, Italy, July 2001.

[22] F. Ye, G. Zhong, S. Lu, and L. Zhang. Energy efficient robust sensing coverage in large sensor networks. Technical report, UCLA, 2002.

[23] F. Ye, G. Zhong, S. Lu, and L. Zhang. Peas: A robust energy conserving protocol for long-lived sensor networks. In The 23nd International Conference on Distributed Computing Systems (ICDCS), 2003.

[24] H. Zhang and J. Hou. On deriving the upper bound of alpha-lifetime for large sensor networks. Technical Report UIUCDCS-R-2004-2410, Department of Computer Science, University of Illinois at Urbana-Champaign, Mar 2004.

[25] H. Zhang and J. C. Hou. Maintaining sensing coverage and connectivity in large sensor networks. Technical Report UIUCDCS-R-2003-2351, Department of Computer Science, University of Illinois at Urbana-Champaign, June 2003.

\section{APPENDIX}

\section{A. RESULTS OF COVERAGE PROCESSES}

For completeness of the paper, we summarize some of the results on asymptotic coverage drawn from [11] that pertain to our derivation in Section 4.

Let the vacancy $V(\ell, \lambda)$ denote the area that is not covered by any node, $S_{i}$ the coverage disk of node $i$, and $\chi(x)$ an indication function of whether a point $x$ is covered by any coverage disk, i.e.,

$$
\chi(x)= \begin{cases}1, & \text { if for all } i, x \notin S_{i}, \\ 0, & \text { otherwise }\end{cases}
$$

When all nodes are randomly placed on the region $R, V$ is a random variable that can be expressed as

$$
V=V(R)=\int_{R} \chi(x) d x .
$$

To calculate the expectation of $V$, we use Fubini's theorem and take the expectation within the integral in Eq. (36). That is,

$$
\begin{aligned}
E(V) & =\int_{R} E(\chi(x)) d x \\
& =\|R\| E(\chi(x)) d x \\
& =\ell^{2} \exp (-\lambda) .
\end{aligned}
$$

We have used interchangeably the terms "complete coverage" and "vacancy area is 0" throughout the paper. This is supported by the following theorem (Theorem 3.3 in [11]).

Theorem 5. Let $C$ be a Boolean model in $R^{k}$ in which covering shapes are distributed as $S$. If $R$ is an open subset of $R^{k}, S$ is a random closed set with $E(\|S\|)<+\infty$, and $V$ is the vacancy area, then

$$
P(V=0 ; R \text { is not completed covered })=0 .
$$

Although the theorem requires $R$ be an open subset, it can be generalized to the case that $R$ is closed and regular. The interested reader is referred to the discussions after the theorem in [11].

\section{B. PROOF OF LEMMA 1}

First we prove (i) $H(k, \alpha)$ monotonically decreases as $k$ increases if $H(k, \alpha)>k$. We need to show that $H(k, \alpha)>$ $H(k+1, \alpha)$. Since we only consider $k$ such that $\alpha>\gamma_{k}$,

$$
\begin{aligned}
& H(k, \alpha)>H(k+1, \alpha) \\
\Leftrightarrow & \frac{\sum_{i=1}^{k-1} i \beta_{i}}{\alpha-\gamma_{k}}>\frac{\sum_{i=1}^{k} i \beta_{i}}{\alpha-\gamma_{k+1}} \\
\Leftrightarrow \quad & \left(\alpha-\gamma_{k+1}\right)\left(\sum_{i=1}^{k-1} i \beta_{i}\right)>\left(\alpha-\gamma_{k}\right)\left(\sum_{i=1}^{k} i \beta_{i}\right) \\
\Leftrightarrow \quad & \left(\gamma_{k}-\gamma_{k+1}\right)\left(\sum_{i=1}^{k-1} i \beta_{i}\right)>k \beta_{k} \alpha-k \gamma_{k} \beta_{k} \\
\Leftrightarrow \quad & \beta_{k}\left(\sum_{i=1}^{k-1} i \beta_{i}\right)>k \beta_{k} \alpha-k \gamma_{k} \beta_{k} \\
\Leftrightarrow & \sum_{i=1}^{k-1} i \beta_{i}>k\left(\alpha-\gamma_{k}\right) \\
\Leftrightarrow \quad & H(k, \alpha)>k .
\end{aligned}
$$

So the first part of the Lemma is proved. In order to prove (ii), we only need to reverse the inequality directions in the above proof. For (iii), we only need to change the inequality sign to equality sign in (i). Next we prove (iv). Since $H(k, \alpha)>k$, we have $\sum_{i=1}^{k-1} i \beta_{i}>k\left(\alpha-\gamma_{k}\right)$. Hence,

$$
\begin{aligned}
H(k+1, \alpha) & =\frac{\sum_{i=1}^{k} i \beta_{i}}{\alpha-\gamma_{k+1}} \\
& =\frac{\sum_{i=1}^{k-1} i \beta_{i}+k \beta_{k}}{\left(\alpha-\gamma_{k}\right)+\beta_{k}} \\
& >\frac{k\left(\alpha-\gamma_{k}\right)+k \beta_{k}}{\left(\alpha-\gamma_{k}\right)+\beta_{k}} \\
& =k .
\end{aligned}
$$

In order to prove (v) and (vi) we only need to change the "> $>$ sign in (iv) to "=" and " $<$ " sign respectively. 
Table 1: Radio transmission range of Berkeley Motes

\begin{tabular}{|c|c|}
\hline Product & Transmission Range \\
\hline MPR300* & $30 \mathrm{~m}$ \\
\hline MPR400CB & $150 \mathrm{~m}$ \\
\hline MPR410CB & $300 \mathrm{~m}$ \\
\hline MPR420CB & $300 \mathrm{~m}$ \\
\hline MPR500CA & $150 \mathrm{~m}$ \\
\hline MPR510CA & $300 \mathrm{~m}$ \\
\hline MPR520CA & $300 \mathrm{~m}$ \\
\hline
\end{tabular}

* MPR300 is the second generation sensors, while the rest are the third generation sensors.

Table 2: Sensing range of several typical sensors

\begin{tabular}{|c|c|c|}
\hline Product & Sensing Range & Typical Applications \\
\hline HMC1002 Magnetometer sensor $[12]$ & $5 \mathrm{~m}$ & Detecting disturbance from automobiles \\
\hline Reflective type photoelectric sensor $[2]$ & $1 \mathrm{~m}$ & Detecting targets of virtually any material \\
\hline Thrubeam type photoelectric sensor $[2]$ & $10 \mathrm{~m}$ & Detecting targets of virtually any material \\
\hline Pyroelectric infrared sensor (RE814S) $[17]$ & $30 \mathrm{~m}$ & Detecting moving objects \\
\hline Acoustic sensor on Berkeley Motes ${ }^{*}[12]$ & $\sim 1 \mathrm{~m}$ & Detecting acoustic sound sources \\
\hline
\end{tabular}

* This result is based on our own measurement on Berkeley motes [12]. 